\title{
20 I 2 Non-invasive assessment of coronary artery disease: a comparison of adenosine stress, studied with contrast echocardiography and 3 Tesla cardiovascular magnetic resonance
}

\author{
Jayanth R Arnold*1, Theodoros D Karamitsos'1, Jane M Francis1, \\ Tammy J Pegg ${ }^{1}$, Nick Searle ${ }^{2}$, Stefan Neubauer ${ }^{1}$, Harald Becher ${ }^{3}$ and \\ Joseph B Selvanayagam ${ }^{1}$
}

Address: ${ }^{1}$ OCMR, University of Oxford, Oxford, UK, ${ }^{2}$ Department of Cardiology, John Radcliffe Hospital, Oxford, UK and ${ }^{3}$ University Department of Cardiovascular Medicine, John Radcliffe Hospital, Oxford, UK

* Corresponding author

from I th Annual SCMR Scientific Sessions

Los Angeles, CA, USA. I-3 February 2008

Published: 22 October 2008

Journal of Cardiovascular Magnetic Resonance 2008, I0(SuppI I):A28I doi:I0.II86/I532-429X-10-SI-A28I

This abstract is available from: http://jcmr-online.com/content/I0/SI/A28I

(c) 2008 Arnold et al; licensee BioMed Central Ltd.

\section{Introduction}

Several modalities are available for the non-invasive assessment of cardiac disease, but MRI and Echocardiography benefit from the absence of ionizing radiation. Myocardial stress perfusion imaging with cardiovascular magnetic resonance (CMR) is now well established in the assessment of coronary artery disease (CAD). It has been demonstrated that a multiparametric approach combining perfusion and infarction imaging further augments the diagnostic performance of CMR [1]. Recent studies indicate that 3 Tesla is the preferred field strength, with increased signal-to-noise and contrast-to-noise ratios compared with 1.5 Tesla [2]. Despite the widespread use of dobutamine stress echocardiography in clinical practice, adenosine stress echocardiography is not routinely used owing to reduced accuracy when wall motion assessment alone is used to evaluate ischaemia. However, the recent advent of second-generation contrast agents now enables a multiparametric approach for echocardiography, involving simultaneous myocardial perfusion and wall motion analysis. We sought to compare two optimized diagnostic strategies in patients with suspected CAD: 1) a combined perfusion and infarct imaging algorithm by CMR at 3 Tesla and 2) combined perfusion and wall motion analysis by adenosine stress echocardiography (SE).

\section{Methods}

Subjects scheduled for elective diagnostic angiography for investigation of exertional chest pain were studied prior to angiography with both SE and CMR. For CMR, patients were studied with first-pass perfusion at 3 Tesla (Trio, Siemens Medical Solutions), at stress $(140 \mathrm{mcg} / \mathrm{kg} / \mathrm{min}$ intravenous adenosine) and at rest. Four short-axis images were acquired every heartbeat using a saturation recovery fast gradient echo sequence and $0.05 \mathrm{mmol} / \mathrm{kg}$ contrast agent (Gadodiamide, Omniscan ${ }^{\mathrm{Tm}}$, GE Healthcare) bolus injection. Perfusion images were acquired every cardiac cycle during the first pass of contrast, using a $\mathrm{T}_{1}$-weighted fast gradient echo sequence (echo time $1.04 \mathrm{~ms}$, repetition time $2 \mathrm{~ms}$, voxel size $\left.2.1 \times 2.6 \times 8 \mathrm{~mm}^{3}\right)$. After rest perfusion, following a further bolus of Gadodiamide (0.045 $\mathrm{mmol} / \mathrm{kg}$ ), delayed enhancement CMR was performed with a T1-weighted segmented inversion-recovery turbo fast low-angle shot (FLASH) sequence (echo time $4.8 \mathrm{~ms}$, voxel size $1.4 \times 2.4 \times 8 \mathrm{~mm}$, flip angle $\left.20^{\circ}\right)$. For the SE study, 2-, 3- and 4-chamber long-axis images were acquired during short breath holds at stress $(140 \mathrm{mcg} / \mathrm{kg} /$ min intravenous adenosine) and at rest, with continuous intravenous infusion of Sonovue (Bracco Diagnostics Inc, Italy). CMR and SE images were interpreted visually by two observers blinded to clinical and angiographic data. Quantitative coronary angiography, performed by a third 
operator blinded to CMR and SE results, served as the reference standard. Significant CAD was defined angiographically as the presence of $\geq 1$ stenosis of $\geq 50 \%$ diameter in any of the main epicardial coronary arteries or their branches with a diameter of $\geq 2 \mathrm{~mm}$.

\section{Results}

Thirty-two subjects were prospectively recruited. One individual did not complete the CMR examination owing to claustrophobia, so 31 subjects were included in the final analysis. The prevalence of CAD was $71 \%$. All CMR and SE images were visually interpretable. Compared to SE, CMR provided higher diagnostic accuracy ( $94 \%$ vs. $81 \%$ ) and sensitivity ( $96 \%$ vs. $78 \%$ ), but similar specificity ( $82 \%$ vs. $82 \%$ ) for detection of significant CAD. CMR also identified disease location with greater sensitivity (LAD $88 \%$ vs. $76 \%$, LCx $75 \%$ vs. $63 \%$, RCA $100 \%$ vs. $62 \%$ ) but similar specificity (LAD 93\% vs. 86\%, LCx 96\% vs. $100 \%$, RCA $89 \%$ vs. $100 \%$ ). However, there was no significant difference in the overall detection of CAD (area under ROC curve: $0.83 \pm 0.08$ SE vs. $0.92 \pm 0.05$ CMR; p $=0.32$, Figure 1 ).

\section{Conclusion}

Whereas the specificity of both techniques is comparable, CMR perfusion imaging provides higher sensitivity by virtue of its high spatial resolution.

\section{References}

I. Klem I, et al: J Am Coll Cardiol 2006, 47: 1630-8.

2. Cheng ASH, et al.: J Am Coll Cardiol 2007, 49:2440-9.

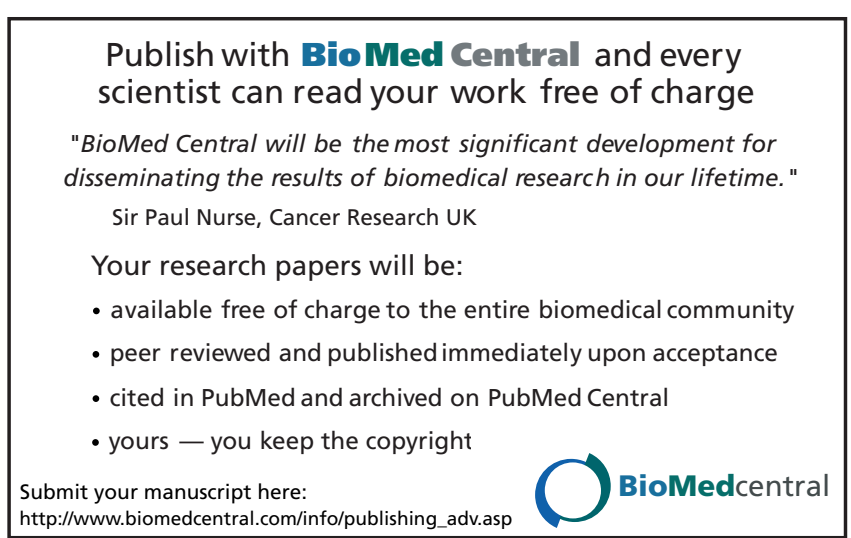

Page 2 of 3

(page number not for citation purposes) 


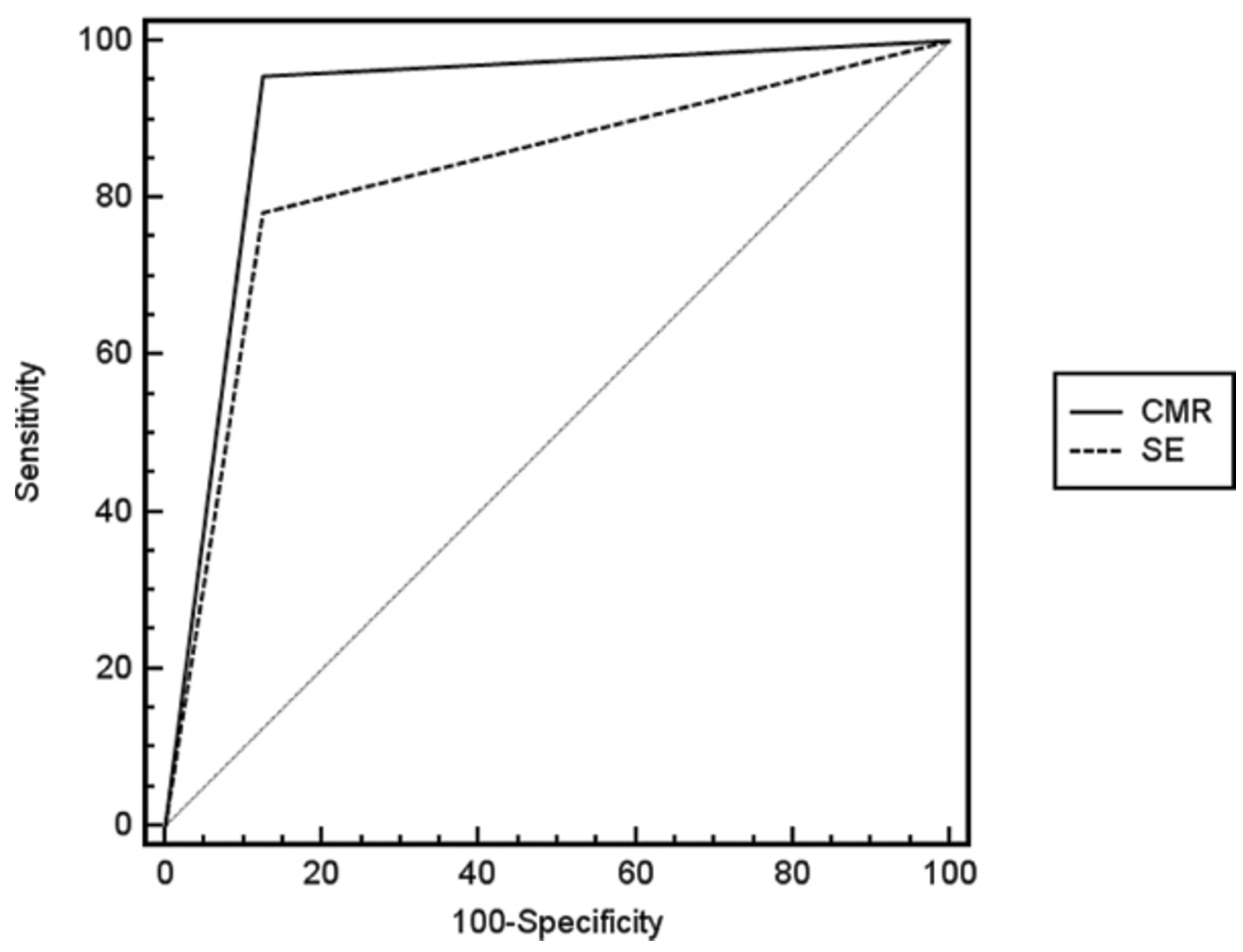

Figure I

ROC curves for CMR and SE for the overall detection of CAD. 